\title{
Von der Entgeltdifferenz zur Entgeltgleichheit der Geschlechter?
}

\begin{abstract}
Angesichts der anhaltend hohen Entgeltlückezwischen den Geschlechtern wird es immer dringlicher, die Ursachen der Ungleichbehandlung aufzudecken - und sie zu beseitigen. Der Staat empfiehlt Unternehmen, die betriebliche Entgeltdifferenz mit dem ökonometrischstatistischen Lohngleichheitsinstrument „Logib-D“"zu analysieren. Ein anderer Weg wird mit dem rechtlich orientierten Instrumentarium „eg-check.de“ eingeschlagen, das im Rahmen eines Forschungsprojektes entwickelt wurde. ${ }^{1}$ Der Beitrag analysiert die Ziele, Möglichkeiten und Grenzen beider Methoden. Es wird gezeigt, dass die Unterbewertung von „Frauenarbeit“ statistisch nicht sichtbar gemacht werden kann. Entgeltgleichheit muss daher unabhängig von der Höhe der betrieblichen Entgeltdifferenz mit Hilfe eines umfassenderen Instrumentes geprüft werden.
\end{abstract}

\section{Einleitung}

In Deutschland beträgt die Entgeltdifferenz zwischen Frauen und Männern 23,2 \% (Statistisches Bundesamt 2010). Sie liegt damit deutlich über dem Durchschnitt der Europäischen Union (18 \%). Mit Besorgnis wird seitens der Politik festgestellt, dass die Entgeltdifferenz hierzulande seit Jahren auf hohem Niveau verharrt und deswegen nun auch auf betrieblicher Ebene vertiefende Analysen und Aktivitäten erforderlich macht, um die Benachteiligung von Frauen beim Entgelt zu erkennen und zu beseitigen. Damit stellt sich in Deutschland erstmalig die Frage, mit welchen Methoden dieses gleichstellungspolitische Ziel erreicht werden kann. Bisher mangelte es an einem konsistenten und umfassenden Prüfinstrument, das den deutschen Rahmenbedingungen gerecht wird. In einigen anderen Ländern bestehen bereits gesetzliche Vorgaben zur Prüfung von Entgeltgleichheit, die auch methodische Vorgehensweisen regeln, z.B. in Schweden oder in der kanadischen Provinz Quebec (vgl. Chicha 2006; Jochmann-Döll 2009); in Österreich werden Unternehmen ab 1.3.2011 gesetzlich zur Einkommenstransparenz verpflichtet. ${ }^{2}$ In Deutschland erfolgt die Prüfung auf freiwilliger Basis. Die aktuelle Diskussion konzentriert sich auf zwei unterschiedliche methodische Ansätze:

Der erste Weg eröffnet sich über die Berechnung der betrieblichen Entgeltdifferenz und - vereinfacht ausgedrückt - die Ermittlung des Anteils, der zu Recht besteht und nicht weiter analysiert wer- den muss, weil Entgeltgleichheit und Gleichbehandlung bei der Beschäftigung gewährleistet sind. Klärungsbedürftig ist der verbleibende Restanteil, der auf Entgeltdiskriminierung zurückzuführen sein kann und näher zu untersuchen ist. Diese Zerlegung der Entgeltdifferenz mittels ökonometrisch-statistischer Regressionsanalysen wird mit dem Lohnmessinstrument „Logib-D“ verfolgt, das die Bundesregierung ${ }^{3}$ Unternehmen zum freiwilligen Selbsttest anbietet. ${ }^{4}$ Mit der Methode soll geprüft werden können, „ob ein Unternehmen Lohngleichheit - d.h. gleicher Lohn für gleichwertige Arbeit - zwischen Frau und Mann gewährleistet oder nicht“, so das Bundesministerium für Familie, Senioren, Frauen und Jugend im März 2009 (BMFSFJ 2009a). Zwischenzeitlich formuliert das BMFSFJ die Zielsetzung allgemeiner: Logib-D „verschafft Informationen über Entgeltgleichheit zwischen Frauen und Männern im Betrieb. ${ }^{\text {"5 }}$

Der zweite Weg verfolgt ebenfalls das Ziel, Entgeltgleichheit zu prüfen. Hierbei wird nicht die betriebliche Entgeltdifferenz zum Ausgangspunkt gewählt, sondern es wird das Entgelt der Geschlechter für jeden Entgeltbestandteil gesondert analysiert (z.B. Grundentgelt, Leistungsvergütung). Geprüft werden mit Hilfe verschiedener Einzelinstrumente nicht nur die betriebliche Entgeltpraxis, sondern auch betriebliche und tarifliche Entgeltregelungen. Für diesen zweiten Zugang steht das Instrument „eg-check.de“ (entgeltgleichheitscheck.de). Es wurde von den Autorinnen entwickelt (vgl. Fußnote 1).

Auf den ersten Blick scheinen beide Methoden dasselbe gleichstellungspolitische Ziel - die Prüfung von Entgelt- gleichheit - zu verfolgen. Doch es lohnt eine genauere Analyse, welches Problemverständnis von Entgelt(un)gleichheit den jeweiligen Prüfmethoden unterliegt. Denn aus den jeweiligen Ergebnissen folgen unterschiedliche handlungsbezogene Konsequenzen. Es geht demnach in diesem Beitrag nicht nur um eine reine Methodendiskussion, sondern um die impliziten Gerechtigkeitsmaßstäbe dieser Methoden und ihre Potenziale zur Erreichung gleichstellungspolitischer Ziele.

Im Folgenden werden beide Prüfinstrumente vorgestellt und ihre jeweiligen konzeptionellen Grundlagen näher

\footnotetext{
1 Dieses Projekt wurde von der Hans-Böckler-Stiftung gefördert. Zu den Ergebnissen vgl. ausführlich Jochmann-Döll/Tondorf 2010. Der eg-check ist verfügbar unter www.eg-check.de.

2 http://www.austria.gv.at/site/cob_41102/ 6595/default.aspx.

3 vgl. hierzu den Koalitionsvertrag der Regierungsparteien CDU/CSU/FDP, 2009, S. 74.

4 Vgl. www.logib-d.de.

5 Vgl. Faltblatt des BMFSFJ (2010).
}

Karin Tondorf, Dr., freiberufliche Wissenschaftlerin und Beraterin zu Entgelt- und Gleichstellungspolitik. Arbeitsschwerpunkte: Modernisierung von Entlohnungssystemen, Leistungs- und zielorientierte Vergütung, Entgeltg/eichheit. e-mail: karintondorf@t-online.de Andrea Jochmann-Döll, Dr., freiberufliche Wissenschaftlerin und Beraterin, GEFA Forschung + Beratung. Arbeitsschwerpunkte: Gleichstellungspolitik, (diskriminierungsfreie) Gestaltung von Entgeltsystemen, Entgeltgleichheit. e-mail: jochmann-doel/@gefa-forschung-beratung.de 
analysiert. Zunächst soll generell geklärt werden, welche Schlussfolgerungen aus der Höhe der betrieblichen Entgeltdifferenz überhaupt gezogen werden können, d.h. ob ein geringer Verdienstunterschied auf geringen Handlungsbedarf in punkto Entgeltgleichheit hindeutet (Abschnitt 2). Abschnitt 3 stellt sodann das Logib-D-Verfahren vor, wobei die These vertreten wird, dass sich über die Ermittlung und Analyse der Entgeltdifferenz mittels Logib-D kein Weg eröffnet, um Entgeltdiskriminierung zu erkennen und zu beseitigen. Das alternative Prüfinstrumentarium, eg-check. de, präsentiert Abschnitt 4 und erläutert zugleich seinen Anwendungsbezug. Abschnitt 5 bilanziert die Vor- und Nachteile bzw. Voraussetzungen beider Verfahren.

\section{Die betriebliche Entgelt- differenz: Was erfasst sie? Was nicht?}

Die Ermittlung und die Analyse der betrieblichen Entgeltdifferenz zwischen Frauen und Männern sind zwei Vorgänge, die unabhängig voneinander vorgenommen werden können. Um Entgeltgleichheit zu prüfen, reicht die Angabe der durchschnittlichen Verdienstunterschiede nicht aus. Denn der Grundsatz der Entgeltgleichheit ist erst dann gewährleistet, wenn Frauen und Männer gleiches Entgelt für eine gleiche oder gleichwertige Arbeit erhalten. Insofern wären bei der Analyse der Entgeltgleichheit arbeitsbezogene Daten einzubeziehen.

Die betriebliche Entgeltdifferenz bezeichnet den prozentualen Unterschied im durchschnittlichen Bruttomonats- bzw. -stundenverdienst ${ }^{6}$ von Arbeitnehmern und Arbeitnehmerinnen. Sie spiegelt die Ist-Situation der Entlohnung wider, die sowohl Ergebnis der geschlechtsbezogenen Personalstruktur als auch der Entgeltpolitik ist. Eine detaillierte Aufschlüsselung dieser Entgeltdifferenz kann innerbetriebliche „Gender Pay Gaps“ in einzelnen Betriebsbereichen und Hierarchieebenen aufzeigen. Eine Aufschlüsselung der Entgeltdifferenz nach Geschlecht, Vollzeit- und Teilzeitbeschäftigten kann zeigen, welcher Teil der Entgeltdifferenz auf unterschiedliche Beschäftigungsverhältnisse zurückzuführen ist. Generell gilt jedoch, dass die Höhe der Entgeltdifferenz noch keine Rückschlüsse auf eine etwaige Entgelt- oder Beschäfti- gungsdiskriminierung von Frauen zulässt. Sie kann auch durch spezifische Merkmale des Geschlechterverhältnisses, wie z.B. hinsichtlich der familiären Arbeitsteilung oder bestimmter Rollenzuschreibungen, verursacht sein, die nach dem Allgemeinen Gleichbehandlungsgesetz (AGG) keine Diskriminierung darstellen.

Insoweit kann auch aus einer unbereinigten Entgeltdifferenz von $0 \%$, wie sie kürzlich für den Öffentlichen Dienst Ostdeutschlands festgestellt wurde (BMFSFJ 2009b, S. 14), nicht gefolgert werden, dass Entgeltgleichheit bereits gewährleistet ist. Zwei fiktive betriebliche Beispiel-Konstellationen zeigen, dass zwischen der Höhe der Entgeltdifferenz und einer potenziellen Entgeltdiskriminierung kein systematischer Zusammenhang besteht:

(1) Beispiel 1: Entgeltdifferenz 20\% - kein Handlungsbedarf: In einem Teilbereich eines Unternehmens erhalten die zwölf dort beschäftigten Männer durchschnittlich $2.500 €$, die 50 beschäftigten Frauen durchschnittlich $2.000 €$. Es besteht also eine Entgeltdifferenz von $20 \%$. Werden die konkreten Tätigkeiten von Frauen und Männern berücksichtigt, zeigt sich Folgendes: sechs Männer und 50 Frauen arbeiten als Kundenberater bzw. -beraterinnen. Sie verrichten die gleiche Arbeit und erhalten einheitlich ein Monatsbruttoentgelt in Höhe von $2.000 €$. Die Entgeltdifferenz beträgt für diese Beschäftigtengruppe $0 \%$ - es besteht kein Handlungsbedarf bezüglich der Entgeltgleichheit, da gleiche Arbeit gleich entlohnt wird. Die Entgeltdifferenz von $20 \%$ entsteht dadurch, dass sechs männliche Führungskräfte beschäftigt sind, die jeweils $3.000 €$ erhalten und dadurch den Durchschnitt erhöhen. Eine Entgeltdiskriminierung ist nicht erkennbar, da die Tätigkeit der Führungskräfte und die Tätigkeit der Kundenberatung nicht gleichwertig sein dürften. Eine Beschäftigungsdiskriminierung ist aus den Daten ebenfalls nicht erkennbar, denn sie zeigen nicht, ob sich eine der Kundenberaterinnen auf eine der Führungspositionen beworben hat und abgelehnt worden ist.

(2) Beispiel 2: Entgeltdifferenz $0 \%$ - Handlungsbedarf: In einem Unternehmen arbeiten 50 weibliche Verkaufskräfte und zehn männliche Lagerarbeiter. Diese Tätigkeiten sind nach dem Tarifvertrag gleich eingruppiert und werden mit $2.500 €$ monatlich vergütet. Die Entgeltdifferenz beträgt so- mit $0 \%$. Es könnte dennoch eine mittelbare Entgeltdiskriminierung bestehen, wenn nämlich die Verkaufstätigkeit unterbewertet wäre, weil zum Beispiel wesentliche Anforderungen psycho-sozialer Art wie Kommunikationsfähigkeit oder wesentliche Belastungen wie ständiges Stehen bei der Arbeitsbewertung nicht berücksichtigt würden. Diese Form der Entgeltdiskriminierung kann statistisch nicht sichtbar gemacht werden. Ob eine Beschäftigungsdiskriminierung vorliegt, ist aufgrund der Daten ebenfalls nicht erkennbar.

Die Beispiele verdeutlichen, dass Entgeltdiskriminierung unabhängig von der Höhe der Entgeltdifferenz bestehen kann. Insbesondere können diese statistischen Werte das Problem der mittelbaren Entgeltdiskriminierung nicht abbilden, da sie nur den Ist-Zustand der Entlohnung widerspiegeln, der ja gerade hinterfragt werden soll. Dies ist umso schwerwiegender, als es sich bei dieser Form der Entgeltdiskriminierung um eine strukturell verankerte Geringschätzung frauendominierter Arbeit handelt, die in Entgeltregelungen ihren Ausdruck findet. Sie ist zwar seit mehr als 50 Jahren verboten, wurde jedoch in der arbeitsweltlichen Wirklichkeit hingenommen und perpetuiert. Das Diskriminierungsverbot blieb faktisch ein „Rechtsprinzip ohne Praxis“ (Winter 2010). Noch heute wird mitunter die Auffassung vertreten, dass die Festsetzung der tariflichen Grundvergütung „mittels einer personenunabhängigen und damit auch geschlechtsneutralen Arbeitsbewertung" erfolge (IW 2009, S. 5; vgl. auch BDA 2010). Da die Entgeltdifferenz nur die gegebene, möglicherweise jedoch diskriminierende Bewertung von Arbeit finanziell widerspiegelt, ist sie als Ausgangsgröße für die Analyse von Entgeltdiskriminierung nicht geeignet.

\section{Die Analyse der Entgelt- differenz mit Logib-D}

Aus den genannten Argumenten folgt, dass für die Analyse von Entgeltgleichheit detaillierte arbeits(platz)bezogene Informationen erforderlich sind, die eine

\footnotetext{
6 Bei den Stundenverdiensten sind die Entgelte von Teilzeit- und Vollzeitbeschäftigten direkt vergleichbar.
} 
Einschätzung erlauben, ob die konkreten Tätigkeiten von Frauen und Männern gleich oder gleichwertig sind. ${ }^{7}$ Nur so kann geprüft werden, ob der Rechtsgrundsatz des gleichen Entgelts für gleiche und gleichwertige Arbeit erfüllt wird. Der Begriff der Gleichwertigkeit verweist auf die Arbeitsbewertung und darin verborgene Entscheidungen über den Wert von „Frauenarbeit" und „Männerarbeit“, die (unbewusst) durch Geschlechterstereotype und Vorurteile geprägt sein können und Benachteiligungen von Frauen bewirken.

Informationen dieser Art werden mit Logib-D jedoch nicht erhoben. Im Zentrum der Basisregression stehen vielmehr, basierend auf der Humankapitaltheorie, persönliche Ausstattungsmerkmale von Männern und Frauen. Konkret werden Ausbildungsjahre, potenzielle Erwerbsjahre und Dienstjahre in die Analyse einbezogen. Entgeltgleichheit gilt dann als gewährleistet, wenn ein Arbeitgeber gleiches Entgelt für die gleichen Voraussetzungen zahlt, die Frauen und Männern hinsichtlich der genannten Merkmale (Humankapitalvariablen) mitbringen. Umgekehrt wird Entgeltdiskriminierung angenommen, wenn Männer und Frauen mit gleicher Humankapitalausstattung ungleich bezahlt werden. Damit wird ein Verständnis von Entgeltgleichheit zugrunde gelegt, das sich vom genannten Rechtsgrundsatz grundlegend unterscheidet.

In der erweiterten Regression werden zusätzlich die Variablen Anforderungsniveau und berufliche Stellung einbezogen, da ein geringeres Entgelt von Frauen nicht allein Ausdruck einer Entgeltdiskriminierung im engeren Sinne (ungleiches Entgelt bei gleichwertiger Humankapitalausstattung), sondern auch einer Beschäftigungsdiskriminierung (ungleiche Stelle bei gleichwertiger Humankapitalausstattung) sein kann. Doch diese arbeitsplatzbezogenen Merkmale werden nicht in Bezug zum Arbeitsentgelt gesetzt, sondern wiederum zu den persönlichen Ausstattungsmerkmalen (Humankapital). Außerdem wird die Zuordnung von Arbeitsplätzen zu Anforderungsniveaus nicht hinterfragt, obwohl sich hierin die mögliche Unterbewertung frauendominierter Arbeit vielfach manifestiert. Auch die zusätzliche Einbeziehung der arbeitsplatzbezogenen Merkmale führt demzufolge nicht dazu, dass mit Logib-D das Prinzip des gleichen Entgelts bei gleicher und gleichwertiger Arbeit überprüft wird.
In der Fachliteratur wird daher hervorgehoben, dass Logib-D Unternehmen Informationen liefere, die zur Aufdeckung von Geschlechtsdiskriminierung nicht geeignet seien. Der Deutsche Juristinnenbund resümiert: „Es kann deshalb die Ziele, die es anstrebt, nicht erreichen" (djb 2010, S. 3). Der Grund hierfür wird in den theoretischen Annahmen von Logib-D verortet. Die konventionellen Humankapitalfaktoren können die geschlechtsbezogenen Entgeltunterschiede nicht hinreichend erklären (Klenner/Ziegler 2010, S. 4). Auch unterstelle die Humankapitaltheorie einen "Qualifikationslohn“, 8 den es in Deutschland nicht gibt (ebd.). Aus rechtlicher Perspektive wird eingewendet, „dass Logib-D mit Prämissen arbeitet, die selbst nicht frei von Diskriminierung sind“ (Winter 2010, S. 332): Personbezogene Merkmale wie Berufsausbildung müssen - so der Rechtsgrundsatz - „für die Ausführung der dem Arbeitnehmer übertragenen spezifischen Aufgaben von Bedeutung sein", entschied der Europäische Gerichtshof. ${ }^{9}$ Das Kriterium Dienstalter berücksichtigt nicht die ungleichen Chancen, die Frauen beim Ansammeln von Betriebszugehörigkeitszeiten haben. Zulässig ist es nach den Maßstäben des europäischen und deutschen Rechts nur dann, wenn mit dem Dienstalter eine größere Berufserfahrung einhergeht und es sich nicht pauschal entgeltsteigernd auswirkt. Jedoch ist auch beim Kriterium der Berufserfahrung genauer hinzusehen, denn nicht immer befähigt sie eine Person dazu, die konkrete Arbeit besser zu verrichten: Bei einfachen Anlerntätigkeiten dürfte eine mehrjährige Berufserfahrung keinen Kompetenzzuwachs erbringen. Wo Berufserfahrung die Qualität der Arbeit steigert, müssten auch Berufsjahre bei anderen Arbeitgebern berücksichtigt werden. Äußern Arbeitnehmerinnen oder Arbeitnehmer Zweifel an der diskriminierungsfreien Verwendung dieses Kriteriums, muss der Arbeitgeber die diskriminierungsfreie Anwendung belegen. ${ }^{10}$ Das Kriterium Potenzielle Erwerbserfahrung berücksichtigt nicht die tatsächlichen Zeiten der Erwerbstätigkeit, sondern nur mögliche (Alter minus sechs Vorschuljahre minus Ausbildungsjahre). Insofern handelt es sich um eine altersabhängige Variable, die einen Verstoß gegen das Allgemeine Gleichbehandlungsgesetz darstellt, da sie Jüngere benachteiligt.

Ein weiterer Kritikpunkt: Logib-D prüft das Bruttoarbeitsentgelt pauschal; differenziert wird lediglich grob zwischen Bruttojahresverdienst und Sonderzahlungen. Dadurch können Diskriminierungsmechanismen, die in einzelnen Entgeltbestandteilen verborgen sind, weder erkannt noch beseitigt werden. Last but not least könnten aus den Ergebnissen von LogibD rechtlich problematische Anpassungsmaßnahmen abgeleitet werden: Wenn Arbeitsentgelte entsprechend dem „Humankapital“ von Frauen und Männern gezahlt werden würden, könnte im Ergebnis gleiche und gleichwertige Arbeit ungleich vergütet werden (Tondorf 2010).

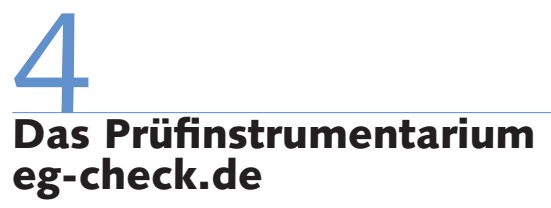

Wenn die beschriebene Feststellung und Analyse der betrieblichen Entgeltdifferenz weder rechtssichere noch ausreichende Informationen zur Verfügung stellt, um Diskriminierungsmechanismen bei der Entgeltfindung eindeutig zu identifizieren, stellt sich die Frage nach einer umfassenderen und auf rechtlichen Grundlagen aufbauenden Prüfmethodik. Das Instrumentarium eg-check.de versteht sich als eine solche Methode und wird nachfolgend mit seinen theoretisch-konzeptionellen Grundlagen vorgestellt.

\subsection{THEORETISCH-KONZEPTIONELLE GRUNDLAGEN}

eg-check.de baut auf Befunden der soziologischen, betriebswirtschaftlichen und rechtswissenschaftlichen Diskriminierungsforschung auf. Bereits in den 1980er Jahren formulierten Vertreterinnen und Vertreter des sogenannten ComparableWorth-Ansatzes die These, dass die Erwerbsarbeit von Frauen systematisch unterbewertet und unterbezahlt werde.

\footnotetext{
7 Diese Argumentation bezieht sich auf den Entgeltbestandteil des anforderungsbezogenen Grundentgelts. Sie gilt jedoch genauso für die Bewertung von Berufserfahrung, Leistung, Erschwernissen oder Überstunden in den entsprechenden Entgeltbestandteilen (Stufensteigerungen, Leistungsvergütung, Erschwerniszuschläge und Überstundenvergütung) und ihrer jeweiligen spezifischen Begründungslogik.

8 Also eine Vergütung nach "mitgebrachter" Qualifikation.

9 Vgl. EuGH vom 17.10.1989-109/88 (Danfoss).
} 10 Vgl. EuGH vom 3.10.2006 C-17/05 (Cadman). 
„Women are paid less because they are in women's jobs, and women's jobs are paid less because they are done by women" (Shepela/Viviano 1984, S. 47). Diese generelle Entwertung von Arbeiten, die von Frauen wahrgenommen werden, wird auch als "evaluative Diskriminierung" bezeichnet (England et al. 2000; Liebeskind 2004). Von der evaluativen Diskriminierung sind auch Männer betroffen, die in typischen Frauenberufen arbeiten. Hiervon zu unterscheiden ist die ,allokative Diskriminierung", die nach Peterson/Saporta (2004) und Peterson/Morgan (1995) dann vorliegt, wenn ,gleich produktive Frauen und Männer auf unterschiedlichen hierarchischen Rängen mit entsprechend unterschiedlicher Entlohnung eingestellt" (Achatz et al. 2005, S. 474) werden.

Die Unterbewertung von Frauentätigkeiten gilt als Folge des „doing gender“ (vgl. West/Zimmerman 1987; Gildemeister/Wetterer 1992). Damit ist gemeint: „a person engaged in virtually any activity may be held accountable for performance of that activity as a woman or a man, and their incumbency in one or the other sex category can be used to legitimate or discredit their other activities" (West/ Zimmerman 1987, S. 136). Geschlechterbezogene Orientierungen, Normen und Differenzen sind nach diesem Verständnis sozial entstanden und werden durch alltägliche Interaktionsprozesse immer wieder neu hervorgebracht. Dasselbe gilt für diskriminierende Praktiken auf dem Arbeitsmarkt und im Betrieb. Geschlechterstereotype spielen eine besondere Rolle beim „doing gender". Es handelt sich hierbei um kulturell weitgehend geteilte Vorstellungsmuster von geschlechterbezogenen Eigenschaften und Verhaltenserwartungen. Sie beschreiben, wie Männer und Frauen sind (deskriptive Dimension), und wie sie sein sollen (präskriptive Dimension) und sie bewerten, was besser und wichtiger ist (evaluative Dimension) (vgl. Knapp 2009). Im Hinblick auf die geschlechterbezogene Zuweisung und Bewertung von Tätigkeiten sorgen Geschlechterstereotype zum einen dafür, dass Frauen aufgrund spezifischer Fähigkeiten und Eigenschaften für bestimmte Tätigkeiten als besonders geeignet gelten. So werden Frauen z.B. als besonders fingerfertig und dadurch als besonders geeignet für Tätigkeiten an Montagebändern und in Nähereien eingeschätzt. Sie gelten außerdem als besonders geduldig, kommunikativ und einfühlsam, deshalb erscheinen sie prädestiniert für soziale und pflegerische Berufe. Geschlechterstereotype prägen zum anderen mit ihrer präskriptiven Dimension die Vorstellungen davon, welche Tätigkeitsfelder für Frauen (und Männer) „angemessen“ und akzeptabel sind und welche nicht. So gilt z.B. der Autohandel noch immer als Männerdomäne, obwohl die für den Autoverkauf erforderlichen Fähigkeiten Frauen keineswegs abgesprochen werden können. Und last but not least beeinflussen Geschlechterstereotype die Bewertung der für bestimmte Tätigkeiten erforderlichen Kenntnisse und Fähigkeiten. So ließe es sich erklären, dass z.B. körperliche Anforderungen und die Verantwortung für die Gesundheit und das Wohlergehen anderer in Pflegeberufen noch keinen Eingang in Eingruppierungstexte finden konnten oder dass psycho-soziale Anforderungen bei der Vergütung eines Arbeitsplatzes bislang, wenn überhaupt, nur in sehr geringem Umfang berücksichtigt werden.

Ridgeway (2001) prägte in diesem Zusammenhang den Begriff der „gender frames“, d.h. geschlechterbezogene Rahmen als immer mitlaufende Hintergrundstrukturierungen, die die Wahrnehmungen, Deutungen und Praxen im Sinne von Vor-Einstellungen beeinflussen. Durch gender-frames und insbesondere "gender status beliefs" werden Tätigkeiten von Frauen und Männern auf der Mikroebene der individuellen Meinungen, Überzeugungen und Selbstverständnisse bestimmte Bedeutungen und Werte zugewiesen. Weiblich konnotierte Tätigkeiten werden aufgrund der geltenden Geschlechterhierarchie quasi automatisch mit geringerem Status und geringerer Wertigkeit assoziiert. Auch auf der Meso-Ebene der kulturellen Ordnungen und gesellschaftlichen und politischen Institutionen sorgen gender frames dafür, dass Tätigkeits- und Praxisfelder geschlechtergetrennt strukturiert werden und dies gleichzeitig in einer Art Zirkelschluss als Beleg dafür dient, dass das jeweilige Geschlecht die für das jeweilige Tätigkeitsfeld erforderlichen Eigenschaften aufweist. Außerdem werden auch die Tätigkeitsfelder mit Wertungen verknüpft, sodass z.B. Erziehungsberufe oder der Sekretariatsbereich als weniger bedeutend gelten als gewerbliche Facharbeit. Auf der Makro-Ebene der Gesellschaft sorgen gender frames und gender status beliefs für eine historische „Trennung und Hie- rarchisierung von Haushalt und Betrieb, von öffentlicher und privater Sphäre, die in unserer Gesellschaft weitreichende Folgen insbesondere für Frauen hat" (Knapp 2009, S. 9). Denn: „Das Zusammenspiel androzentrischer Traditionen und kapitalistischer Verwertungsmaximen lässt alle Tätigkeiten und Praxisfelder, die sich nicht nach den Maßstäben der kapitalistischen Zeitökonomie rationalisieren lassen, als weniger relevant erscheinen“ (ebd.).

Den noch fehlenden Schlussstein zwischen den stereotypen Bewertungen und Hierarchisierungen durch gender frames und gender status beliefs einerseits und der vorgefundenen Entgeltpolitik und -praxis auf tariflicher wie betrieblicher Ebene andererseits kann der Institutionen-Ansatz beisteuern (Krüger 2001). Mit ihm wurde nicht nur darauf verwiesen, dass in sozialen Institutionen wie Familie, Bildungssektor oder Arbeitsmarkt jeweils unterschiedliche Konstruktionen von Geschlecht vorherrschen können, sondern sie unterstellen auch jeweils bestimmte Geschlechterverhältnisse und richten ihre Regeln und Normen nach diesen Geschlechterbildern aus. Im Hinblick auf die soziale Institution tariflich oder betrieblich vereinbarter Entgeltsysteme ist festzustellen: Viele der Regelungen zur Vergütung von Teilzeitkräften bzw. zur Bewertung von Tätigkeiten, die überwiegend in Teilzeit ausgeübt werden, dürften auf die ungebrochene Existenz des „Ernährer-HinzuverdienerinModells" zugeschnitten sein. Ein weiteres Beispiel sind die äußerst geringen VollzeitVerdienste in frauendominierten Tätigkeitsbereichen wie Friseurin oder (Zahn-, Tier-) Arzthelferin, die nur begrenzt eine eigenständige ökonomische Existenz ermöglichen. Auch einige Leistungskriterien bei der leistungsbezogenen Vergütung z.B. zeitliche Flexibilität, Mobilität - basieren auf der Vorstellung eines (männlichen) Beschäftigten, der ohne familiäre oder reproduktionsbezogene Verpflichtungen frei und quasi unbegrenzt seine Leistung erbringen kann.

Die hier vorgestellten Ursachenanalysen führen zu der Schlussfolgerung, dass ein maßgeblicher Diskriminierungsmechanismus in der Unterbewertung von Arbeit liegt. Für Deutschland wiesen Rohmert und Rutenfranz bereits 1975 in einem arbeitswissenschaftlichen Gutachten für die damalige Bundesregierung darauf hin, dass die Verfahren der Arbeitsbewertung nicht diskriminierungsfrei sind (Rohmert/ 
Rutenfranz 1975). ${ }^{11}$ In der Schweiz wurden in staatlichem Auftrag geschlechtsneutrale Arbeitsbewertungssysteme entwickelt. ${ }^{12}$ In Großbritannien schufen Tarifparteien des kommunalen Bereichs ein diskriminierungsfreieres Arbeitsbewertungsverfahren (NJC). ${ }^{13}$ Empirische Belege für die Unterbewertung einer Reihe von weiblich dominierten Tätigkeiten lieferten für Deutschland die Studie von Krell et al. (2001) am Beispiel von Beschäftigten der Stadt Hannover, die Untersuchung von Arbeitsplätzen an Hochschulen von Stefaniak et al. (2002) sowie die vergleichende Analyse von Betrieben der Chemie- und Metallindustrie durch Carl/Krehnke (2004). Während sich diese Studien auf die Grundentgeltdifferenzierung bezogen, wiesen Bevan/ Thompson (1992), Fried et al. (2000) sowie Tondorf/Jochmann-Döll (2005) auf Diskriminierungspotenziale bei der Leistungsvergütung hin.

Für das gleichstellungspolitische Handeln folgt daraus, dass die Prozesse und Ergebnisse der Bewertung von Arbeit - und im weiteren Sinne auch der Bewertung von Mehrarbeit, Leistung, Berufserfahrung, Erschwernissen an Frauen- und Männerarbeitsplätzen - einer Diskriminierungsprüfung zu unterziehen sind. Entsprechend fundierte Analysen wie eg-check.de schließen am Rechtsgrundsatz des gleichen Entgelts für gleiche und gleichwertige Arbeit an. Für Betriebs- und Tarifparteien ist nur dieses Problemverständnis von Entgeltdiskriminierung verbindlich und nur hierauf können Beschäftigte ihre Ansprüche stützen.

\subsection{GRUNDSÄTZE, VERFAHREN UND INSTRUMENTE VON EG-CHECK.DE}

eg-check.de versteht sich als modulares arbeitsbezogenes Analyseverfahren, mit dem Entgeltdiskriminierung aufgrund des Geschlechts geprüft und beseitigt werden kann. Angeboten werden verschiedene Einzelinstrumente im Sinne eines „Werkzeugkastens“, wobei die einzelnen „Werkzeuge“ für unterschiedliche Entgeltbestandteile konstruiert sind und für unterschiedliche Prüfsituationen genutzt werden können. Im Unterschied zu dem in Abschnitt 3 dargestellten ökonomischen Prüfkonzept orientiert sich eg-check.de an folgenden Grundsätzen:

(1) Rechtliche Prüflogik: Das Instrumentarium orientiert sich am Rechtsgrundsatz des gleichen Entgelts für gleiche und gleichwertige Arbeit. Nach diesem „Gerechtigkeitsmaßstab" sind die konkrete auszuübende Arbeit von Frauen und Männern und das dafür gezahlte Entgelt Gegenstände der Prüfung.

(2) Separate Prüfung jedes Entgeltbestandteils: Die gesonderte Prüfung ist rechtlich

\section{Abb. 1: Instrumente des eg-check.de und prüfbare Entgeltbestandteile}

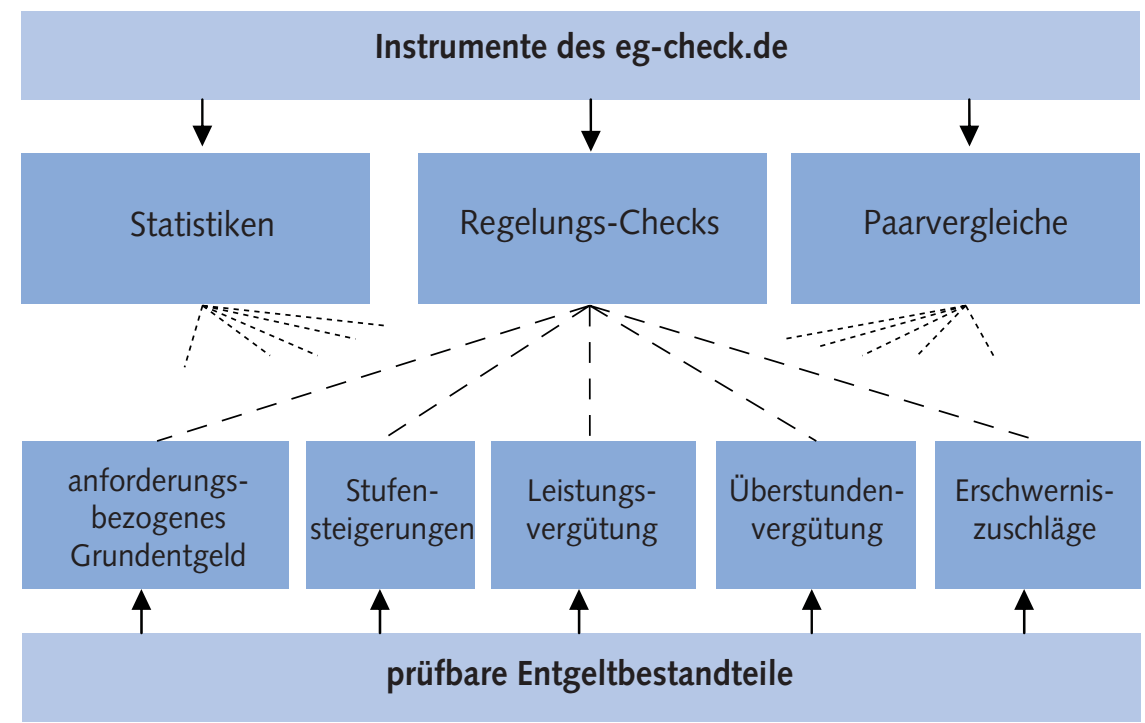

geboten $^{14}$ und auch inhaltlich notwendig: Denn jeder Entgeltbestandteil folgt einer eigenen Begründungs- und Berechnungslogik, die diskriminierende Potenziale enthalten kann.

(3) Prüfung unmittelbarer und mittelbarer Diskriminierung: Da Entgeltdiskriminierung in zwei Formen vorkommen kann, muss das Analyseinstrument beide Formen sichtbar machen können. Mittelbare Entgeltdiskriminierung ist nach rechtlicher Definition durch „dem Anschein nach neutrale Vorschriften, Kriterien oder Verfahren“ ( $\$ 3$ Ziffer 2 AGG) verursacht; daher müssen auch betriebliche, tarifliche und gesetzliche Entgeltregelungen geprüft werden.

(4) Prüfung von der aggregierten bis zur individuellen Ebene: Aggregierte Daten können erste Hinweise auf Entgeltdiskriminierung ergeben. Da diese letztlich Individuen betrifft, erstreckt sich die Prüfung auch auf die Einzelfallebene.

\section{INSTRUMENTE UND PRÜFBARE ENTGELTBESTANDTEILE}

Mit eg-check.de stehen drei Arten von Instrumenten zur Verfügung: Statistiken, Regelungs-Checks und Paarvergleiche. Sie können zur Prüfung von derzeit fünf Entgeltbestandteilen genutzt werden: für das anforderungsbezogene Grundentgelt, Stufensteigerungen beim Grundentgelt, Leistungsvergütung, Überstundenvergütung und Erschwerniszuschläge (Abbildung 1).

Statistiken (direkte Vergleiche) enthalten anonymisierte Entgeltdaten, welche nach Geschlecht und weiteren spezifischen Kriterien differenzieren, die für den jeweiligen Entgeltbestandteil charakteristisch sind.

Regelungs-Checks bestehen aus Fragen und Erläuterungen, mit deren Hilfe Dis-

11 Das Gutachten wurde im Jahr 1988 durch das BAC (vom 27.04.1998 - AZR 707/97) als Grundlage der sog. geänderten Verkehrsanschauung der körperlichen Schwere von Arbeiten anerkannt.

12 Abakaba (Analytische Bewertung von Arbeitstätigkeiten nach Katz und Baitsch: Katz/Baitsch 1996.

13 NJC = Arbeitsbewertungssystem für die lokalen Behörden im Vereinigten Königreich, das von einem nationalen, von Arbeitgebern und Gewerkschaften gemeinsam besetzten Ausschuss (National Joint Council) entwickelt wurde.

14 Vgl. u.a. EuGH vom 17.5.1990, Rs. C-262/88 (Barber). 
kriminierungspotenziale in betrieblichen Vereinbarungen und Tarifverträgen systematisch aufgespürt werden können.

Paarvergleiche dienen der Prüfung der Entgeltgleichheit auf der individuellen Ebene, d.h. zwischen einer einzelnen weiblichen und einem einzelnen männlichen Beschäftigten. Die für den Paarvergleich herangezogenen Vergleichsaspekte unterscheiden sich je nach Entgeltbestandteil.

\section{ZIELGRUPPEN, ANWENDUNGS- SZENARIEN UND EINSTIEGSWEGE}

Das Prüfinstrumentarium eg-check.de richtet sich nicht nur an Arbeitgeber, sondern kann auch von Betriebs- und Personalräten, Gleichstellungsbeauftragten und Tarifparteien genutzt werden. Darüber hinaus kann eg-check.de für alle diejenigen hilfreich sein, die Beschäftigte und Arbeitgeber in Fragen der Entgeltgleichheit informieren und beraten, also für Interessenverbände sowie Rechtsanwälte und Rechtsanwältinnen. Arbeitsrichter und -richterinnen können eg-check.de im Rahmen von Entgeltgleichheitsklagen heranziehen. Auch Beschäftigte können einzelne Instrumente nutzen, soweit ihnen die Daten und Informationen zur Verfügung stehen.

Das Instrumentarium eg-check.de kann je nach Ausgangssituation und Prüfinteresse genutzt werden. Es ist nicht erforderlich, alle Entgeltbestandteile zu prüfen oder alle Beschäftigten in ein Prüfprojekt einzubeziehen. Dementsprechend ist es auch nicht zwingend, dass alle Prüfinstrumente zum Einsatz kommen. Je nach konkretem Prüfinteresse kann die Analyse unterschiedlich zeitaufwändig sein.

Eine betriebliche Prüfung der Entgeltgleichheit sollte im Idealfall gemeinsam von Arbeitgeber- und Arbeitnehmerseite im Rahmen eines Projektes vorgenommen werden. Sinnvoll ist ein Projektplan, der durch eine paritätisch besetzte Projektgruppe erstellt werden sollte (vgl. dazu ausführlich Tondorf/Jochmann-Döll 2010, S. 134).

Übersicht 1 fasst die wesentlichen Unterschiede zwischen Logib-D und egcheck.de überblicksartig zusammen.

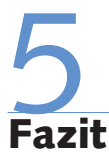

Es wurde gezeigt, dass weder eine unbereinigte noch eine mittels Logib-D bereinigte betriebliche Entgeltdifferenz das Ausmaß von Entgeltdiskriminierung aufzeigen kann. Entgeltgleichheit ist unabhängig von der Höhe der Entgeltdifferenz zu prüfen, denn es besteht kein systematischer $\mathrm{Zu}$ sammenhang zwischen der Höhe der Entgeltdifferenz und Entgeltdiskriminierung. Die statistischen Ergebnisse bilden nur die Resultate von möglicherweise diskriminierenden Bewertungsentscheidungen ab. Werden diese als gegeben akzeptiert, ist die Unterbewertung der Arbeit als bedeutendes Problem der Entgeltdiskriminierung nicht mehr im Blickfeld der weiteren Analysen.

Es dürfte deutlich geworden sein, dass die Prüfung von Entgeltdiskriminierung komplex ist und nicht mit einem einfachen Mausklick bewerkstelligt werden kann. Dies liegt vor allem daran, dass sich die Entlohnung selbst zu einem komplexen Gebilde mit vielfältigen Bestandteilen, Begründungskriterien und Regeln entwickelt hat. Ein Analyseinstrument muss dieser Komplexität folgen. Dass dies für die Akteure und Akteurinnen auf der betrieblichen oder der tariflichen Ebene beträchtliche Anstrengungen bedeutet, soll an dieser Stelle keineswegs verschwiegen werden. Schwierigkeiten dürften zum einen in der Datenverfügbarkeit und -zugänglichkeit liegen, zum anderen aber auch in der immer noch weit verbreiteten Unkenntnis über und mangelnden Sensibilität für Diskriminierungsmechanismen beim Entgelt.

Da sich die Auswahl einer Prüfmethode nach dem anvisierten gleichstellungspolitischen Ziel richtet, wäre auf betrieblicher Ebene diesbezüglich Klarheit zu schaffen. Je nach Ziel gilt:

(1) Soll ein erster Überblick über die betriebliche Entgeltsituation gewonnen werden, bringt die Ermittlung der Entgeltdifferenz zwischen Frauen und Männern nähere Informationen. Dies lässt jedoch noch keinerlei Aufschlüsse über mögliche Diskriminierungen beim Entgelt oder bei der Beschäftigung zu, d.h. die Verdienstdifferenzen können in vollem Umfang, teilweise oder gar nicht sachlich gerechtfertigt sein.

(2) Bezieht sich das Ziel auf eine gleichstellungsorientierte Personalpolitik oder im engeren Sinne auf eine Erhöhung des Frauenanteils in Führungspositionen oder in männerdominierten Bereichen, kann eine Analyse mit Logib-D erste Hinweise auf einen nicht qualifikationsgerechten Personaleinsatz von Frauen geben. Allerdings wird die persönliche Schul- und Berufsausbildung bei Logib-D nur in Jahren betrachtet, d.h. die inhaltliche Ausrichtung der Ausbildung sowie zusätzliche Fortbildungen bleiben unberücksichtigt.

(3) Soll geprüft werden, inwieweit Einstellungen, Aufstiege, Weiterbildung sowie Arbeits- und Entlassungsbedingungen diskriminierungsfrei erfolgen, sind

\begin{tabular}{|c|c|c|}
\hline & Logib-D & eg-check.de \\
\hline \multicolumn{3}{|l|}{ Aspekte } \\
\hline Ziel & Geschlechterbezogene Darstellung der Entgeltstruktur & Prüfung von Entgeltgleichheit \\
\hline Grundlage & Ökonomisch: Humankapitaltheorie & $\begin{array}{l}\text { Rechtlich: } \\
\text { AGG und Unionsrecht }\end{array}$ \\
\hline Definition von Entgeltgleichheit & $\begin{array}{l}\text { Gleiches Entgelt bei gleicher Ausstattung mit persönlichen } \\
\text { Ausstattungsmerkmalen (Humankapital) }\end{array}$ & Gleiches Entgelt bei gleicher und gleichwertiger Arbeit \\
\hline Prüfmethode & Regressionsanalysen & Statistiken, Regelungs-Checks, Paarvergleiche \\
\hline Prüfung des Arbeitsentgelts & $\begin{array}{l}\text { Pauschal: regelmäßiges Bruttomonatsentgelt und unregelmäßige } \\
\text { Bestandteile }\end{array}$ & Jeder Entgeltbestandteil separat \\
\hline Aussagekraft der Ergebnisse & $\begin{array}{l}\text { Aussagen über die Zusammensetzung der betrieblichen } \\
\text { Entgeltlücke }\end{array}$ & $\begin{array}{l}\text { Aussagen über } \\
\text { - die Höhe von Benachteiligungen } \\
\text { - diskriminierende Elemente in Regelungen } \\
\text { - Ursachen der Benachteiligung }\end{array}$ \\
\hline
\end{tabular}


entsprechend gestaltete Statistiken, Regelungs-Checks und Paarvergleiche geeignet (vgl. hierzu Ganser et al. 2011).

(4) Besteht das Ziel darin, Entgeltgleichheit zu prüfen und geschlechtsbezogene Diskriminierungen zu beseitigen, können diesbezügliche Informationen mittels egcheck.de gewonnen werden. Prüfinstrumente, die auf Annahmen der Humankapitaltheorie basieren, eignen sich nicht für die Anwendung auf betrieblicher Ebene, da sie weder mit dem Gleichstellungsrecht noch mit den empirischen Grundsätzen der Entlohnung, insbesondere der anforderungs- oder leistungsbezogenen Entgeltdifferenzierung, kompatibel sind. Ihre Ergebnisse sind daher für die Durchsetzung von Entgeltgleichheit nicht verwendbar.

Zum gegenwärtigen Zeitpunkt beruht die Prüfung von Entgeltgleichheit in Deutschland auf dem Prinzip der Freiwilligkeit. Möglicherweise erkennen gleichstellungsorientierte Arbeitgeber den Nutzen eines ernsthaften Bemühens um „Fair p(l)ay“ bei der Entlohnung und werten es als wichtiges Signal für (potenzielle) Aktionäre, Kunden und Bewerberinnen bzw. Bewerber sowie als Teilelement einer motivierenden Unternehmenskultur. Wenn eine freiwillige Prüfung jedoch nicht auf breiter Ebene erfolgt - was aufgrund der bisherigen Erfahrungen mit freiwilligen Vereinbarungen wahrscheinlich ist-, wird ein Gesetz erforderlich, das die Prüfung und Beseitigung von Entgeltdiskriminierung mit wirkungsvollen Instrumenten verbindlich regelt.

\section{LITERATUR}

Achatz, J./Gartner, H./Glück, T. (2005): Bonus oder Bias? Mechanismen geschlechtsspezifischer Entlohnung, in: Kölner Zeitschrift für Soziologie und Sozialpsychologie 57 (3), S. 466-493

Bevan, S./Thompson, M. (1992): Merit Pay, Performance Appraisal, and Attitudes to Women's Work, Final Report of a study by the Institute of Employment Studies for the Equal Opportunities Commission, IMS Report (234), Brighton

Bundesministerium für Familie, Senioren, Frauen und Jugend (BMFSFJ) (Hrsg.) (2009a): Lohngleichheitsinstrument Bund für Deutschland - Logib-D, Stand März 2009, Berlin

Bundesministerium für Familie, Senioren, Frauen und Jugend (BMFSFJ) (2009b): Der Verdienstunterschied von Frauen und Männern im öffentlichen Bereich und in der Privatwirtschaft, Berlin

Bundesministerium für Familie, Senioren, Frauen und Jugend

(BMFSFJ) (Hrsg.) (2010): Logib-D. Lohngleichheit im Betrieb Deutschland, Stand März 2010, Berlin

Bundesvereinigung der Deutschen Arbeitgeberverbände (BDA) (2010): Lohnunterschiede/Equal Pay. Von Diskriminierung kann keine Rede sein, http://www.arbeitgeber.de/www/arbeitgeber.nsf/id/EFBF4 53CCE70D855C12574F000345727? open \& ccm =800, download vom 13. August 2010

Carl, A.-H./Krehnke, A. (2004): Geschlechterdiskriminierung bei der betrieblichen Grundentgeltdifferenzierung: Positionen und Perspektiven von Management, Betriebsrat und Beschäftigten, Wiesbaden

CDU/CSU/FDP (2009): Wachstum. Bildung. Zusammenhalt. Koalitionsvertrag zur 17. Legislaturperiode, Berlin

Chicha, M.T. (2006): A comparative analysis of promoting pay equity: models and impacts, International Labour Office, ILO Working Paper (49), Genf

Deutscher Juristinnenbund (djb) (2010): Logib-D: Verfehlte Hoffnungen auf Anzeige diskriminierungsverdächtiger Entgeltdifferenzen, Stellungnahme vom 9. März 2010, http://www.djb.de/Kom/K1/st10-3/, download vom 23. August 2010

England, P./Hermsen. J.M./Cotter, D.A. (2000): The Devaluation of Women's Work: A Comment on Tam, in: Amercian Journal of Sociology 105 (6), S. $1741-1751$
Fried, A./Wetzel, R./Baitsch, C. (2000): Wenn zwei das Gleiche tun.... Diskriminierungsfreie Personalbeurteilung, hrsg. vom Eidgenössischen Büro für die Gleichstellung von Frau und Mann, Zürich Ganser, P./Jerchel, K./Jochmann-Döll, A./Tondorf, K. (2011): PraxisHandbuch Gleichbehandlung, Hamburg (im Erscheinen) Gildemeister, R./Wetterer, A. (1992): Wie Geschlechter gemacht werden. Die soziale Konstruktion der Zwei-Geschlechtlichkeit und ihre Reifizierung in der Frauenforschung, in: Knapp, G.A./Wetterer, A. (Hrsg.): Traditionen-Brüche. Entwicklungen feministischer Theorie, Freiburg i.Br. Institut der deutschen Wirtschaft (IW) (Hrsg.) (2009): Stellungnahme zur öffentlichen Anhörung des Ausschusses für Familie, Senioren, Frauen und Gesundheit des Deutschen Bundestages am 28. Januar 2009, Ausschussdrucksache 16(13)419h zu BT-Drs. 16/11175, 16/11192, 16/8784 Jochmann-Döll, A. (2009): Entgeltgleichheit herstellen. Erfahrungen aus anderen Ländern nutzen, in: Arbeitsrecht im Betrieb 30 (12), S. 688-691

Jochmann-Döll, A./Tondorf, K. (2010): Entgeltgleichheit prüfen mit eg-check.de, hrsg. von der Hans-Böckler-Stiftung, Arbeitspapier (214), Düsseldorf

Katz, C./Baitsch, C. (1996): Lohngleichheit für die Praxis. Zwei Instrumente zur geschlechtsunabhängigen Arbeitsbewertung, hrsg. vom Eidgenössischen Büro für die Gleichstellung von Frau und Mann, Zürich Klenner, C./Ziegler, A. (2010): Mit Logib-D zur Überwindung der geschlechtsspezifischen Entgeltlücke?, WSI Report (03), Düsseldorf Knapp, G.-A. (2009): Geschlechterungleichheiten im Betrieb und in der Gesellschaft, Dokumentation eines Vortrags anlässlich der 5. Gleichstellungstagung der Hans- Böckler-Stiftung am 1./2. Oktober 2009 in Berlin, http://www.boeckler.de/pdf/v_2009_01_10_knapp.pdf Krell, G./Carl, A.-H./Krehnke, A. (2001): Diskriminierungsfreie Bewertung von (Dienstleistungs-)Arbeit. Ein Projekt im Auftrag der Gewerkschaft Öffentliche Dienste, Transport und Verkehr, hrsg. vom Bundesvorstand ver.di - Vereinte Dienstleistungsgewerkschaft, Stuttgart Krüger, H. (2001): Gesellschaftsanalyse: der Institutionenansatz in der Geschlechterforschung, in: Knapp, G.-A./Wetterer, A. (Hrsg.): Soziale Verortung der Geschlechter. Gesellschaftstheorie und feministische Kritik, Münster, S. 63-90 
Liebeskind, U. (2004): Arbeitsmarktsegregation und Einkommen - vom Wert "weiblicher" Arbeit, in: Kölner Zeitschrift für Soziologie und Sozialpsychologie 56 (4), S. 630-652

Peterson, T./Morgan, L.A. (1995): Separate and unequal: Occupation Establishment Sex Segregation and the Gender Wage Gap, in: American Journal of Sociology 101 (2), S. 329-365

Peterson, T./Saporta, I. (2004): The Opportunity Structure for Discrimination, in: American Journal of Sociology 109 (4), S. 852-901 Ridgeway, C.L. (2001): Interaktion und die Hartnäckigkeit der Geschlechter - Ungleichheit in der Arbeitswelt, in: Heintz, B. (Hrsg.): Geschlechtersoziologie, Sonderheft 41 der Kölner Zeitschrift für Soziologie und Sozialpsychologie, Opladen, S. 250-275

Rohmert, W./Rutenfranz, J. (1975): Arbeitswissenschaftliche Beurteilung der Belastung und Beanspruchung an unterschiedlichen industriellen Arbeitsplätzen, hrsg. vom Bundesministerium für Arbeit und Sozialordnung, Bonn

Shepela, S. T./Viviano, A. T. (1984): Some psychological Factors Affecting Job Segregation and Wages, in: Remick, H. (Hrsg.): Comparable Worth and Wage Discrimination, Philadelphia, S. 47-58
Statistisches Bundesamt (2010): Pressemitteilung 079 vom 5.3., Berlin Stefaniak, A./Tondorf, K./Kühnlein, G./Webster, J./Ranftl, E. (2002): "Alles, was recht ist“. Entgeltgleichheit durch diskriminierungsfreie Arbeitsbewertung in Deutschland, Großbritannien und Österreich. Ergebnisse eines Forschungsprojekts, München/Mering

Tondorf, K. (2010): Betriebliche Prüfung von Lohngleichheit - mit Logib-D?, in: Der Betrieb 63 (11), S. 616-617

Tondorf, K./Jochmann-Döll, A. (2005): (Geschlechter-)Gerechte Leistungsvergütung? Vom (Durch-) Bruch des Leistungsprinzips in der Entlohnung, Hamburg

West, C./Zimmerman, D. H. (1987): Doing Gender, in: Gender and Society 1 (2), S. 125-151

Winter, R. (2010): Diskriminierungsfreie(re) Entgeltgestaltung - leider noch immer ein Prinzip ohne Praxis, in: Hohmann-Dennhardt, C./Körner, M./Zimmer, R. (Hrsg.): Geschlechtergerechtigkeit. Festschrift für Heide Pfarr, Baden-Baden, S. 320-333 